\title{
A New Centralized Sensor Fusion-Tracking Methodology Based on Particle Filtering for Power-Aware Systems
}

\author{
Yan Zhai, Member, IEEE, Mark B. Yeary, Senior Member, IEEE, \\ Joseph P. Havlicek, Senior Member, IEEE, and Guoliang Fan, Senior Member, IEEE
}

\begin{abstract}
In this paper, we address the problem of target tracking in a collaborative acoustic sensor network. To cope with the inherent characteristics and constraints of wireless sensor networks, we present a novel target-tracking algorithm with power-aware concerns. The underlying tracking methodology is described as a multiple-sensor tracking/fusion technique based on particle filtering. As discussed in the most recent literature, particle filtering is defined as an emerging Monte Carlo state estimation technique with proven superior performance in many target-tracking applications. More specifically, in our proposed method, each activated sensor transmits the received acoustic intensity and the direction of arrival (DOA) of the target to the sensor fusion center (a dedicated computing and storage platform, such as a microserver). The fusion center uses each received DOA to generate a set of estimations based on the state partition technique, as described later in this paper. In addition, a set of sensor weights is calculated based on the acoustic intensity received by each activated sensor. Next, the weighted sum of the estimates is used to generate the proposal distribution in the particle filter for sensor fusion. This technique renders a more accurate proposal distribution and, hence, yields more precise and robust estimations of the target using fewer samples than those of the traditional bootstrap filter. In addition, since the majority of the signal processing efficiently resides on the fusion center, the computation load at the sensor nodes is limited, which is desirable for power-aware systems. Last, the performance of the new tracking algorithm in various tracking scenarios is thoroughly studied and compared with standard tracking methods. As shown in the theory and demonstrated by our experimental results, the state-partition-based centralized particle filter reliably outperforms the traditional method in all experiments.
\end{abstract}

Index Terms-Monte Carlo methods, multisensor systems, sequential estimation, state estimation, tracking.

\section{INTRODUCTION}

D URING recent years, with the dramatic advances in the design of microelectromechanical systems and ad hoc

Manuscript received July 15, 2006; revised January 25, 2008. This work was supported in part by a Grant from the National Oceanic and Atmospheric Administration under the NWRT to MPAR initiative and by the U.S. Army Research Laboratory and the U.S. Army Research Office under Grant W911NF04-1-0221.

Y. Zhai is with Schlumberger Technology Center, Sugar Land, TX 77478 USA (e-mail: yan.zhai@ieee.org).

M. B. Yeary and J. P. Havlicek are with School of Electrical and Computer Engineering, The University of Oklahoma, Norman, OK 73019-0631 USA (e-mail: yeary@ou.edu; joebob@ou.edu).

G. Fan is with School of Electrical and Computer Engineering, Oklahoma State University, Stillwater, OK 74078 USA (e-mail: glfan@okstate.edu).

Color versions of one or more of the figures in this paper are available online at http://ieeexplore.ieee.org.

Digital Object Identifier 10.1109/TIM.2008.919009 networking protocols, there has been an emerging trend toward the use of sophisticated wireless networks of unattended sensor devices for a variety of new monitoring and control applications [1]-[3]. Sensor networks provide the monitoring for the physical world via many distributed wireless sensing devices that are deployed in the region of interest. Each of these low-cost tiny devices, which are also known as a sensor node, has limited processing and communication capabilities with a limited power supply. The sensing information is collaboratively processed between the sensor nodes and at the sensor fusion center (e.g., microserver), which has significantly greater bandwidth, computation, and energy capabilities compared to sensor nodes. Locating and tracking moving stimuli or targets are the primary tasks for a sensor network in many practical applications, such as robot navigation, security surveillance, and battlefield awareness [1]. In this paper, we consider tracking a ground vehicle in a wireless acoustic sensor network using directions of arrival (DOAs) as the measurements. The sensor network that is studied here consists of a large number of sensor nodes and one mobile sensor fusion center, which has access to the information gathered by the sensor nodes and is installed on a monitoring vehicle. The tracking scenario is illustrated in Fig. 1. For tracking targets in a sensor network, it is important to design a sequential method that can dynamically fuse the information that is sent by multiple sensors without requiring significant processing capabilities at the sensor nodes and excessive communication within the network. The selection of the data routing scheme and the communication protocol is out of the scope of this study.

This paper is based on our previous work [4] with the addition of more detailed derivation, more experimental work and analysis, and more current references in this field. This paper is organized as follows. In Section II, we briefly review the work of target tracking in a sensor network. Section III analyzes the mathematical model for target tracking in an acoustic sensor network. The new tracking algorithm is formulated in Section IV. Last, simulation results and conclusion remarks are provided in Sections V and VI, respectively.

\section{RELATED WORK IN SENSOR NETWORK TARGET TRACKING}

The earliest work of target tracking using multiple sensors involves various Kalman-filter-based methods, such as the extended Kalman filter (EKF), the interacting multiple model 


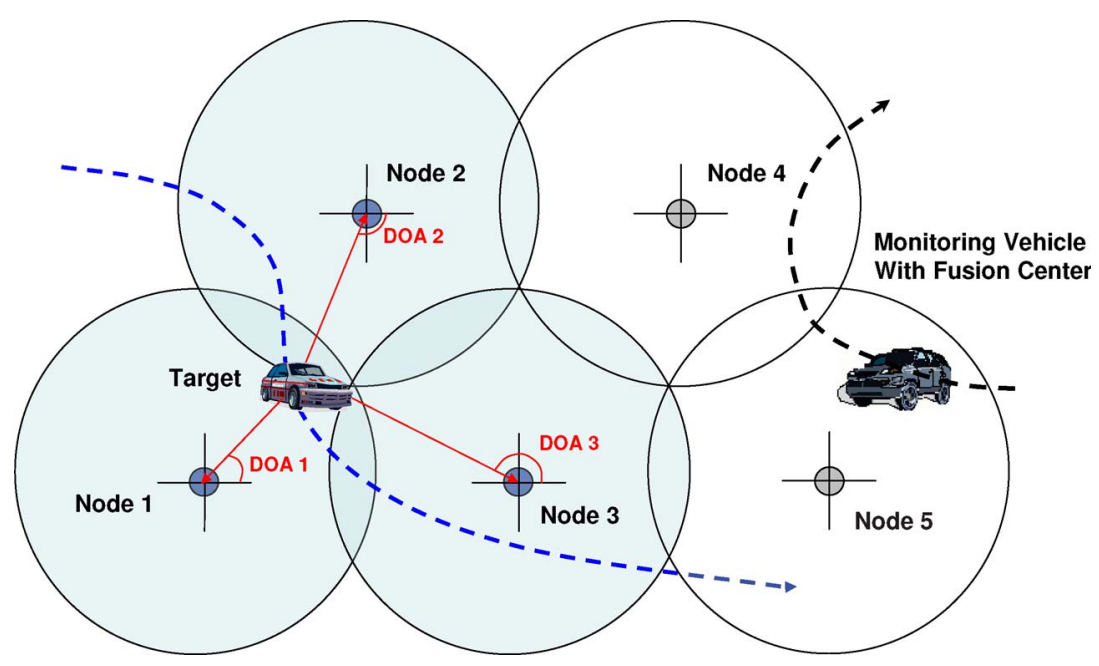

Fig. 1. Target tracking in a wireless sensor network. Sensor nodes 1-3 are in the active mode, and sensor nodes 4 and 5 are in the sleep mode. The fusion center is installed on the monitoring vehicle, which has the access to all sensor nodes.

(IMM) EKF, and the probability data association methods [5]. Later, the hidden-Markov-model-based methods were studied. In [6], an IMM tracking algorithm is developed based on discretized target state space. Meanwhile, the "theory of evidence" is used in [6] to construct the observation model. In addition, a maximum-likelihood source localization method based on acoustic sensor readings was also proposed for target tracking in sensor networks [7]. However, as indicated in [8], this method is sensitive to the parameter perturbation, and the computational complexity is high for a multitarget location estimation. Recently proposed methods also include the "dynamic convoy tree" target tracking [9] as well as others. More recently, particle filter (PF) based methods have been receiving increased attention. For example, a PF tracking method is developed in [8], in which a simple form of particle filtering, known as the bootstrap filter, is used, and its particle weights are calculated based on the acoustic energy readings. However, it is well known that a bootstrap filter takes the state transition prior as the proposal distribution, which does not take into account the current measurements-it simply relies on one-step-ahead predictions. When the likelihood distribution is narrow with respect to the transition prior distribution, many particles will receive negligible weights, which means that abundance of computation will be wasted. In [10], a new PF tracking algorithm is proposed with an improved Gaussian proposal and a more complex measurement model.

In general, PF tracking algorithms are always categorized as centralized or decentralized. In [8] and [10], there are examples of centralized versions. Centralized particle filters (CPFs) imply that all signal processing tasks are carried out at the fusion center. On the other hand, various decentralized (or distributed) particle filter (DPF) tracking algorithms are discussed in the current literature. In [11], a DPF algorithm is proposed; however, this algorithm demands significant communication in the sensor network to update the complete particle set and involves complicated learning procedures. In [13], the sensors are divided into disjoint "cliques," and the PFs that are associated with each clique are ran in parallel.
Following this, a Gaussian mixture model is used to approximate the whole particle set. Although this strategy does not require significant communication between sensor nodes, it requires computationally intensive filtering algorithms to be implemented at the sensor node, whose computation capability and power supply are very limited in many applications. Last, the work in [13] depends on many sensors to be turned on to achieve their desired performance. In general, many tracking methods that are discussed here either require the sensor nodes to have substantial signal processing capabilities or require a more complex sensor network architecture. For example, the recent work of [9] requires an elaborate multinode structure that includes multiple "lead nodes" to track the target. To rectify these problems, we propose a new PF tracking algorithm that is based on the state partition technique and parallel EKFs (SP-PEKFs). As demonstrated in our simulation results, the new algorithm renders considerably accurate tracking results while requiring only a small amount of communication protocols in the network and limited processing tasks at each sensor node, which, in turn, reduces power consumption.

\section{PROBLEM Formulation}

Here, we describe the tracking problem in two stages. The first introduces the sensor model, whereas the second discusses the dynamic model of the moving target.

\section{A. Acoustic Wave Intensity Decay Model}

We consider a distributed wireless acoustic sensor network, which is composed of deterministically or randomly deployed sensors, whose positions are known to the fusion center, which is either stationary or mobile. The sensor locations could be computed by using the methods proposed in [14]-[17] or using an onboard Global Positioning System. An acoustic sensor is capable of detecting the DOA of a perambulating target when the target comes into the neighborhood (i.e., the effective range) of this sensor. A sensor has limited computing capabilities to 
calculate the received acoustic intensity, and it also serves as a transmitter/receiver. We assume that the sensor nodes are dense enough such that during each sampling period, there is at least one sensor in the active mode. Assuming that the acoustic source can be treated as a point target, and the sound propagation is isotropic, the acoustic intensity that is received by sensor nodes can be modeled as follows [1], [22], [23]:

$$
P(t)=\frac{S(t)}{\|X(t)-r(t)\|^{\frac{\alpha}{2}}}+\varepsilon(t)
$$

where $S(t)$ denotes the acoustic intensity emitted from the source (target) that is located at $X(t)$, and $P(t)$ represents the acoustic intensity received by a sensor that is located at $r(t)$. The variable $\alpha$ is an attenuation coefficient, and $\varepsilon(t)$ is the additive noise that is assumed to have a zero-mean Gaussian distribution. In the sensor network, the sensors are designed to switch to their active mode when the received acoustic intensity is above a certain threshold. Once a sensor is in the active mode, the acoustic intensity that is received by the sensor will be calculated and sent to the fusion center. At the fusion center, a set of sensor weights is calculated as $\mathcal{W}_{k}(t)=$ $P_{k}(t) /\left(\sum_{k=1}^{K} P_{k}(t)\right)$, where $\mathcal{W}_{k}(t)$ denotes the sensor weight for sensor $k, K$ is the total number of the active sensors, and $P_{k}(t)$ is the acoustic intensity that is received by sensor $k$.

\section{B. Target Dynamic Model}

In this paper, we focus on tracking a moving target using a bearings-only measurement (i.e., DOA angle). As the target moves through the network field, sensors that are located in the neighborhood of the target will be turned on and will send out the DOA of the target to the sensor fusion center. For each single activated sensor, the state space model of a targettracking problem is formulated here. The target is represented by a vector given as follows:

$$
\mathbf{x}_{t}=\left[x(t) \quad y(t) \quad v_{x}(t) \quad v_{y}(t)\right]^{T}
$$

where $(x(t), y(t))$ is the target location in a local Cartesian coordinate, which takes the sensor location as its origin. The variables $v_{x}(t)$ and $v_{y}(t)$ denote the target velocities along the $x$-axis and $y$-axis, respectively. The discrete time model for the kinematics of a moving target is given as follows [26]:

$$
\mathbf{x}_{t}=\mathbf{F} \cdot \mathbf{x}_{t-1}+\Gamma \cdot \mathbf{v}_{t-1}
$$

where

$$
\mathbf{F}=\left[\begin{array}{llll}
1 & 0 & T & 0 \\
0 & 1 & 0 & T \\
0 & 0 & 1 & 0 \\
0 & 0 & 0 & 1
\end{array}\right], \quad \Gamma=\left[\begin{array}{cc}
T^{2} / 2 & 0 \\
0 & T^{2} / 2 \\
T & 0 \\
0 & T
\end{array}\right]
$$

where $T$ is the sampling rate. The process noise $\mathbf{v}_{t}$ is a $2 \times 1$ noise vector with a distribution $\mathbf{v}_{t} \sim \mathcal{N}\left(\mathbf{0}, \mathbf{Q}_{v}\right)$, where

$$
\mathbf{Q}_{v}=\left[\begin{array}{cc}
\sigma_{v}^{2} & 0 \\
0 & \sigma_{v}^{2}
\end{array}\right]
$$

The measurement of the tracking system is the angle between the current sensor (the coordinate origin) and the location of the target, which is given by

$$
z(k)=\arctan \left(\frac{x(t)}{y(t)}\right)+w(t)
$$

where the scalar variable $w(t)$ denotes the observation noise that is distributed as $w \sim \mathcal{N}\left(0, \sigma_{w}^{2}\right)$. Equations (3) and (4) constitute the state space model for the bearings-only tracking problem, in which the observation equation contains nonlinearities. In addition, the velocities $v_{x}(t)$ and $v_{y}(t)$ are the hidden states of the system, which do not have direct measurements.

\section{POWER-AWARE PF FOR TARGET TRACKING IN SENSOR NETWORKS}

\section{A. New Scheme for Tracking in Sensor Networks}

As indicated in Section III, the sensor nodes in the network have both limited computation and communication capabilities. In addition, it is difficult or even impossible to frequently replenish the power supply in many sensor networks. All of these constraints pose new challenges for the target-tracking problem. On the other hand, particle filtering has emerged to be a promising tracking algorithm in various applications. The design of the proper proposal distribution is crucial to implement a PF. This distribution governs the weights of the particles that approximate the posterior distribution. In light of this, we propose a novel particle filtering algorithm with its proposal that is generated from a weighted sum of estimates calculated by using each sensor feedback. More specifically, at the fusion center, the DOAs returned from each activated sensor are used to formulate a set of individual trackers. These trackers can generate fast but perhaps not very accurate estimates. However, these estimates should remain in the neighborhood of the true target location. In addition, a set of sensor weights $\mathcal{W}_{k}(t)$ is calculated by using the acoustic intensity that is received by each active sensor node. Then, a weighted sum is calculated based on these estimates and the sensor weights. Last, by taking the weighted sum estimations as the proposal distribution, a $\mathrm{PF}$ is applied for target sensor fusion. Here, in this paper, we propose to use the state partition technique with a bank of EKFs (SP-PEKFs) to generate individual trackers using each received DOA. The SP-PEKF method is discussed in Section IV-B.

\section{B. Method of the SP-PEKF}

The SP-PEKF technique [24], [25] is a method to estimate the statistics of the states from a nonlinear system given as follows:

$$
\begin{aligned}
& \mathbf{x}(t)=f(\mathbf{x}(t-1))+\mathbf{v}(t-1) \\
& \mathbf{y}(t)=h(\mathbf{x}(t))+\mathbf{w}(t)
\end{aligned}
$$

The rationale behind this method is to generate a set of samples $x_{i}, i=1, \ldots, N$ associated with each state $x$ according to a given initial state distribution $\mathcal{N}(x(0), R(0))$. Then, these 
samples are partitioned and propagated through a bank of EKFs. Last, the estimated state is given by the weighted sum of these samples. More specifically, each state of the nonlinear system, or each component of $\mathbf{x}(t)$, is partitioned as follows:

$$
x(t) \triangleq x_{n}(t)+x_{r}(t)
$$

where $x_{n}(t)$ and $x_{r}(t)$ denote the nominal part and the residual part of the true state, respectively. The samples $x_{i}(0)$ are generated according to the initial conditions as follows:

$$
\begin{aligned}
x(0) & =x_{n_{i}}(0)+x_{r_{i}}(0) \\
\hat{x}(0) & =\hat{x}_{n_{i}}(0)+\hat{x}_{r_{i}}(0) \\
R(0) & =R_{n_{i}}(0)+R_{r_{i}}(0) \\
\hat{x}_{n_{i}}(0) & =\hat{x}(0), \quad R_{n_{i}}(0)=R(0) \\
\hat{x}_{r_{i}}(0) & =0, \quad R_{r_{i}}(0)=0 .
\end{aligned}
$$

The variables $R_{n_{i}}$ and $R_{r_{i}}$ represent the covariance that is associated with the nominal state and the residual state, respectively. In the case when $\hat{x}(0)$ and $R(0)$ are known, the nominal state can be viewed as a deterministic variable propagating through the system as follows:

$$
x_{n_{i}}(t)=f\left(x_{n_{i}}(t-1)\right)+v_{n_{i}}(t-1)
$$

where $v_{n_{i}}(t)$ has the same distribution as $v(t)$, but $v_{n_{i}}(t)$ is a different realization. Following that, the system is linearized about $x_{n_{i}}(t)$ as

$$
\begin{aligned}
x(t) & \approx f\left(x_{n_{i}}(t-1)\right)+F\left(x_{n_{i}}(t-1)\right) \cdot\left[x(t-1)-x_{n_{i}}(t-1)\right]+v(t-1) \\
& =f\left(x_{n_{i}}(t-1)\right)+F\left(x_{n_{i}}(t-1)\right) \cdot x_{r_{i}}(t-1)+v(t-1)
\end{aligned}
$$

where

$$
F\left(x_{n_{i}}(t)\right)=\left.\frac{\partial(f(x))}{\partial x}\right|_{x=x_{n_{i}}(t)} .
$$

The above equation can be simplified as

$$
x_{r_{i}}(t) \approx F\left(x_{n_{i}}(t-1)\right) \cdot x_{r_{i}}(t-1)+v(t-1)-v_{n_{i}}(t-1)
$$

where $F$ is the Jacobian (see [24] and [25] for details). Furthermore, the following equation is constructed in a similar way:

$$
y(t) \approx h\left(x_{n_{i}}(t)\right)+H\left(x_{n_{i}}(t)\right) \cdot x_{r_{i}}(t)+w(t)
$$

where

$$
H\left(x_{n_{i}}(t)\right)=\left.\frac{\partial(h(x))}{\partial x}\right|_{x=x_{n_{i}}(t)} .
$$

Equations (12) and (13) form an approximate dynamic model to the nonlinear system that is given by (5). This approximate model is linear to the residual part of the state $x_{r_{i}}(t)$. A bank of EKFs is then applied to update $x_{r_{i}}(t)$. In the context of our problem statement, a summary of the nonlinear filtering technique based on the SP-PEKF is described below. For further information on SP-PEKFs, see [24] and [25].
To begin, the one-step-ahead prediction of the residual state $\hat{x}_{r_{i}}(t \mid t-1)$ and the filtered estimate of $\hat{x}_{r_{i}}(t \mid t)$ are given by

$$
\begin{aligned}
\hat{x}_{r_{i}}(t \mid t-1) & =F\left(x_{n_{i}}(t-1)\right) \cdot \hat{x}_{r_{i}}(t-1 \mid t-1)-v_{n_{i}}(t-1) \\
\hat{x}_{r_{i}}(t \mid t) & =\hat{x}_{r_{i}}(t \mid t-1)+G_{i}(t) \cdot \hat{y}_{i}(t \mid t-1)
\end{aligned}
$$

where $G_{i}(t)$ is the $i$ th subfilter gain given as

$$
G_{i}(t)=R_{i}(t \mid t-1) H\left(x_{n_{i}}(t)\right)^{T} R_{y_{i}}(t \mid t-1)^{-1} .
$$

The residual state prediction covariance and its update covariance are given by

$$
\begin{aligned}
R_{r_{i}}(t \mid t-1)= & R_{i}(t \mid t-1) \\
= & F\left(x_{n_{i}}(t-1)\right) R_{i}(t-1 \mid t-1) \\
& \times F\left(x_{n_{i}}(t-1)\right)^{T}+\sigma_{v}^{2} \\
R_{r_{i}}(t \mid t)= & R_{i}(t \mid t) \\
= & {\left[I-G_{i}(t) H\left(x_{n_{i}}(t)\right)\right] R_{i}(t \mid t-1) . }
\end{aligned}
$$

The pseudo-innovation process and its covariance are given by

$$
\begin{aligned}
\hat{y}_{i}(t \mid t-1) & \approx y(t)-h\left(\hat{x}_{i}(t \mid t-1)\right) \\
R_{y_{i}}(t \mid t-1) & =H\left(x_{n_{i}}(t)\right) R_{i}(t \mid t-1) \times H\left(x_{n_{i}}(t)\right)^{T}+\sigma_{w}^{2} .
\end{aligned}
$$

The variables $\sigma_{v}^{2}$ and $\sigma_{w}^{2}$ denote the variance of the process noise and of the observation noise, respectively. The estimation of the state at time $t$ is given by the following weighted sum:

$$
\hat{x}_{\text {total }}(t \mid t)=\sum_{i=1}^{N} \hat{x}_{i}(t \mid t) \cdot \psi_{i}(t)
$$

where $\hat{x}_{i}(t \mid t)=x_{n_{i}}(t)+\hat{x}_{r_{i}}(t \mid t)$. The weight for each subfilter is given as follows:

$$
\psi_{i}(t)=\frac{L_{i}(t \mid t) \cdot \psi_{i}(t-1)}{\sum_{i=1}^{N} L_{i}(t \mid t) \cdot \psi_{i}(t-1)}
$$

where

$$
\begin{aligned}
L_{i}(t \mid t)=\mid R_{y_{i}}(t \mid t & -1)\left.\right|^{-0.5} \\
& \times \exp \left[-\frac{1}{2}\left\|\hat{y}_{i}(t \mid t-1)\right\|^{2} \cdot R_{y_{i}}^{-1}(t \mid t-1)\right] .
\end{aligned}
$$

Last, the overall estimation error covariance $R_{\text {total }}$ is given by

$$
\begin{aligned}
& R_{\text {total }}(t \mid t)=\sum_{i=1}^{N}\left\{R_{i}(t \mid t)\right.+\left[\hat{x}(t \mid t)-\hat{x}_{i}(t \mid t)\right] \\
&\left.\cdot\left[\hat{x}(t \mid t)-\hat{x}_{i}(t \mid t)\right]^{T}\right\} \cdot \psi_{i}(t) .
\end{aligned}
$$

The above filtering method is based on the idea of using a bank of EKFs to generate multiple state trajectories to simulate the true state trajectory. The weighted sum gives the estimation of the system state, and the weights are adaptively updated as time evolves. 


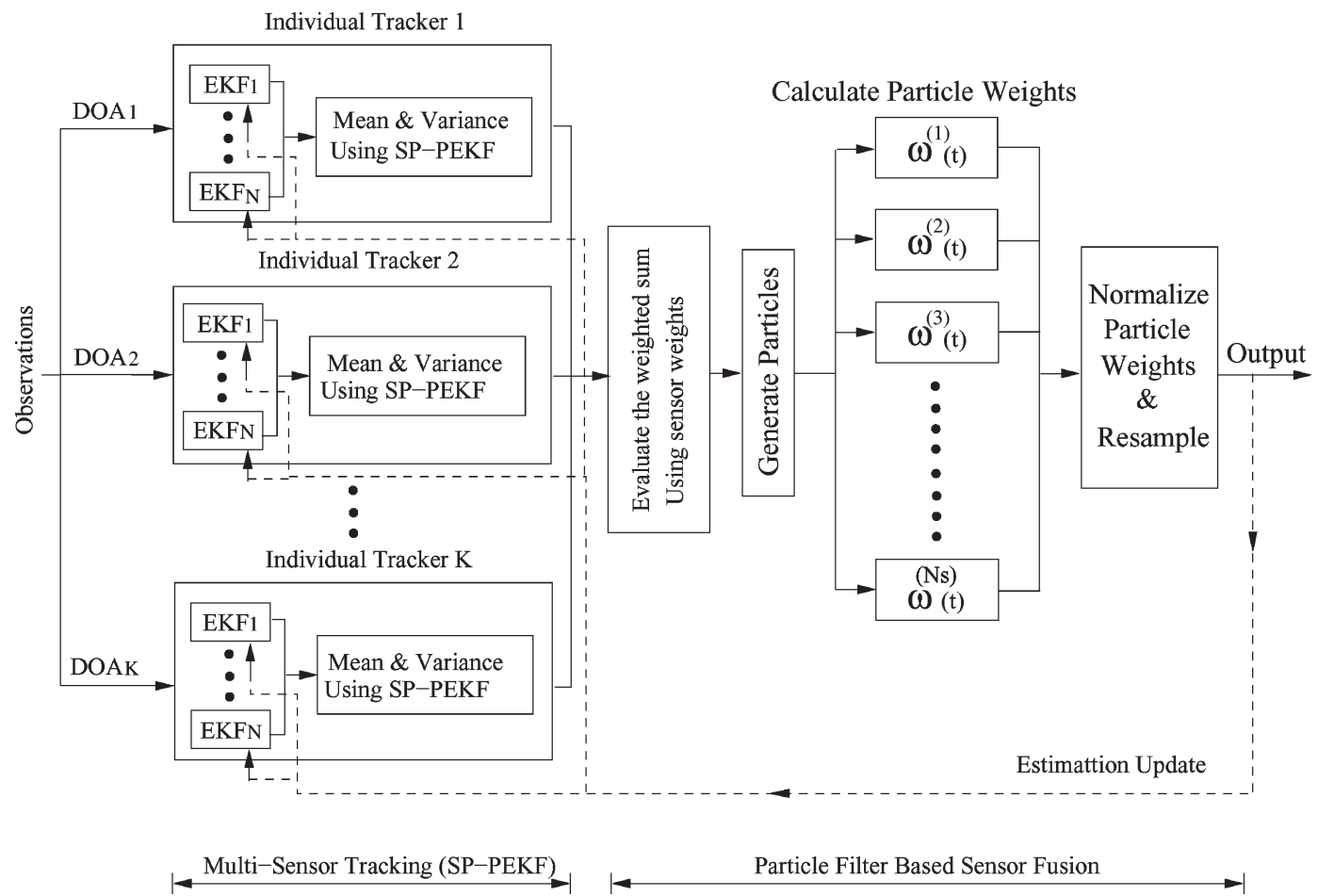

Fig. 2. PF-based sensor tracking/fusion with a power-aware design.

\section{PF-Based Sensor Network Tracking}

In the past few years, particle filtering has received considerable attention in the engineering literature [18]-[21] as the next-generation nonlinear filtering technique, whose objective is to estimate the posterior distribution of the dynamic system states $p(\mathbf{x}(0: t) \mid \mathbf{y}(1: t))$. Particle filtering is a sequential Monte Carlo method based on the concept of importance sampling and Bayesian theory. By applying the sequential importance sampling, the posterior distribution can be approximated by a set of weighted samples shown as follows:

$p(\mathbf{x}(0: t) \mid \mathbf{y}(1: t)) \approx \sum_{i=1}^{N_{s}} \tilde{\omega}^{(i)}(t) \delta\left(\mathbf{x}(0: t)-\mathbf{x}^{(i)}(0: t)\right)$

where $N_{s}$ denotes the number of particles (samples). The variable $\tilde{\omega}^{(i)}(t)$ is called the normalized importance weight and is given by

$$
\tilde{\omega}^{(i)}(t)=\frac{\omega^{(i)}(t)}{\sum_{j=1}^{N s} \omega^{(j)}(t)}
$$

where

$$
\omega^{(i)}(t)=\omega^{(i)}(t-1) \frac{p\left(\mathbf{y}(t) \mid \mathbf{x}^{(i)}(t)\right) p\left(\boldsymbol{x}^{(i)}(t) \mid \mathbf{x}^{(i)}(t-1)\right)}{q\left(\mathbf{x}^{(i)}(t) \mid \mathbf{x}^{(i)}(0: t-1), \mathbf{y}(1: t)\right)} .
$$

The variable $\omega^{(i)}(t)$ denotes the importance weight that is associated with the $i$ th particle at time $t$. The particles (samples), which are denoted by $\mathbf{x}^{(i)}(t)$, are drawn from the proposal distribution

$$
\mathbf{x}^{(i)}(t) \sim q\left(\mathbf{x}(t) \mid \mathbf{x}^{(i)}(0: t-1), \mathbf{y}(1: t)\right) .
$$

As mentioned earlier, we choose the PF proposal as the weighted sum of estimations calculated from the SP-PEKF using each returned DOA and sensor weight. More specifically, in each iteration, the individual tracker generates an estimate with mean and variance given by (21) and (23). Then, a weighted sum is calculated using the sensor weights. Next, a PF algorithm is applied by using the weighted sum as the proposal for sensor fusion. Last, the estimates are updated for the next iteration. Our new multiple-sensor PF-SP-PEKF algorithm is illustrated in Fig. 2 and is summarized in Algorithm 1.

Estimate $x(t)$ according to $x(t) \approx\left(1 / N_{s}\right) \sum_{i=1}^{N_{s}} x^{i \star}(t)$, and update the proposal.

We also provide two additional remarks as follows. First, to take advantage of the estimates that are derived from the PF, the particles are recursively fed back into the SP-PEKF at the end of each iteration. This update stage serves as a "correction" step for the SP-PEKF at each interaction. Second, we typically choose the number of filter bank channels $N$ in (21)-(23) to be much smaller than the number of particles $N_{s}$. Our experience is that taking $10 \leq N \leq 20$ is generally sufficient to obtain significantly improved performance compared to a bootstrap filter.

\section{Data Fusion and Tracking Results}

Extensive experiments have been conducted to test the accuracy and the robustness of the new proposed algorithm. The experimental results are presented in this section. To ascertain the effectiveness of the new algorithm (SP-CPF), we first apply it to a single-sensor tracking problem (i.e., tracking an aircraft using a single radar with DOA measurements) and compare it with other known tracking techniques. Next, we examine the application of the SP-CPF for ground vehicle tracking in an 


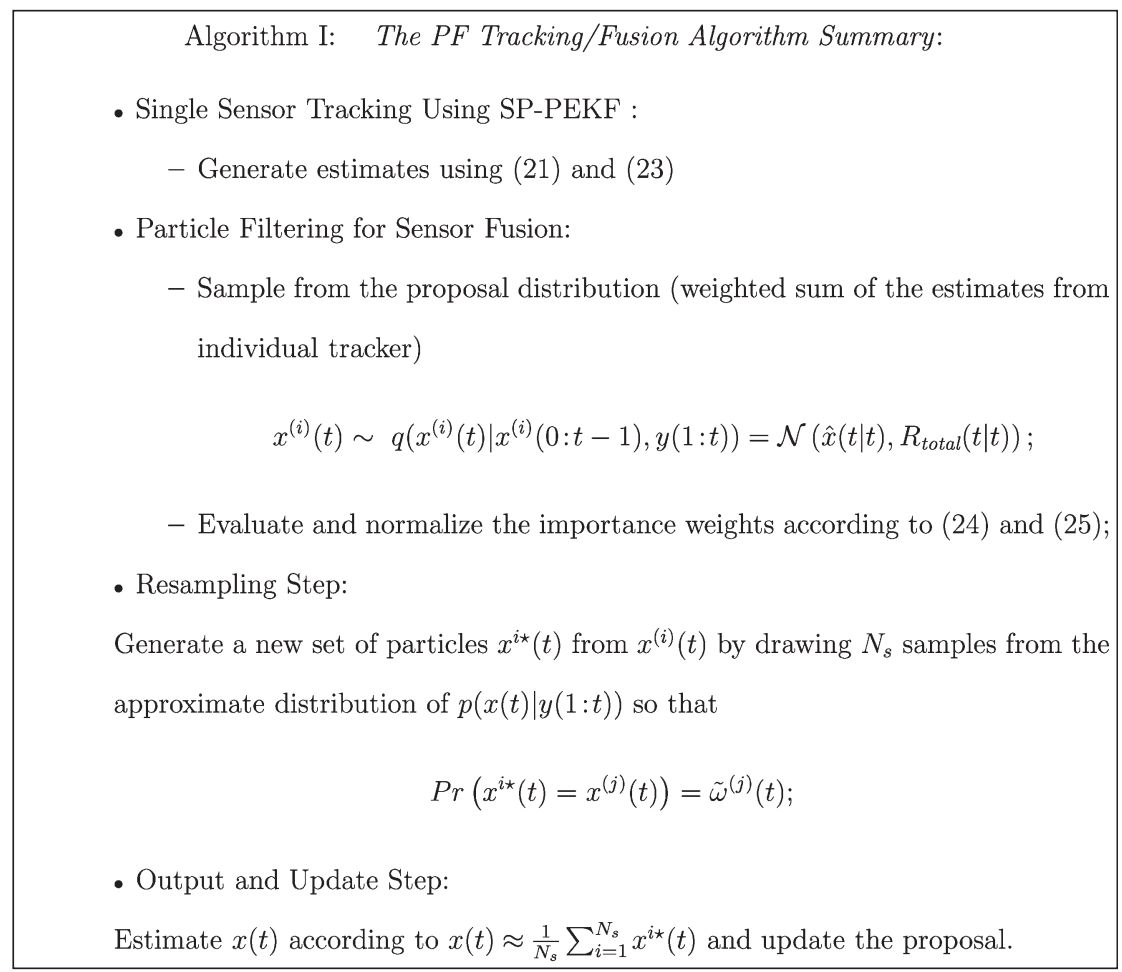

acoustic sensor network for three different tracking scenarios: 1) a rectilinear motion that is disturbed by random noise; 2) a motion that involves sharp coordinate turns with periodically changing velocities and accelerations; 3) a dual-mode motion trajectory that is initially described by a constant acceleration and then followed by a constant deceleration. Based on our research, these three motion models are the major components that represent the true motions of a ground vehicle.

Now, we study the single-sensor tracking problem. The data in this experiment show the air traffic at a major airport (Oklahoma City International Airport), as measured by the National Weather Radar Testbed in Norman, OK. These data were taken on September 2005. Here, the detection was made within a $90^{\circ}$ sector, looking toward the airport, and the origin establishes the location of the radar. We apply three tracking algorithms to a specific path. These algorithms are the EKF, the traditional PF (bootstrap), and the single-sensor SP-CPF. Fig. 3 depicts the experimental results of one typical realization of the tracking algorithm. As indicated in the figure, our new algorithm gives better estimates of the target locations, as compared to the EKF and the bootstrap filter. This experimental result is important because tracking based on single DOA measurements has traditionally been a difficult task [26]. Next, we consider tracking scenarios with multiple sensors.

\section{A. Tracking Scenario Number 1}

In this tracking scenario, we analyze tracking a target, which has a rectilinear motion that is disturbed by random noise. The target trajectory together with a grid sensor topology is shown in Fig. 4(a). In all the simulations in this study, we assume that the moving target, i.e., a ground vehicle, has a noise level of $70 \mathrm{~dB}$. The sensor threshold is set to $72 \%, 75 \%$, and $78 \%$ of

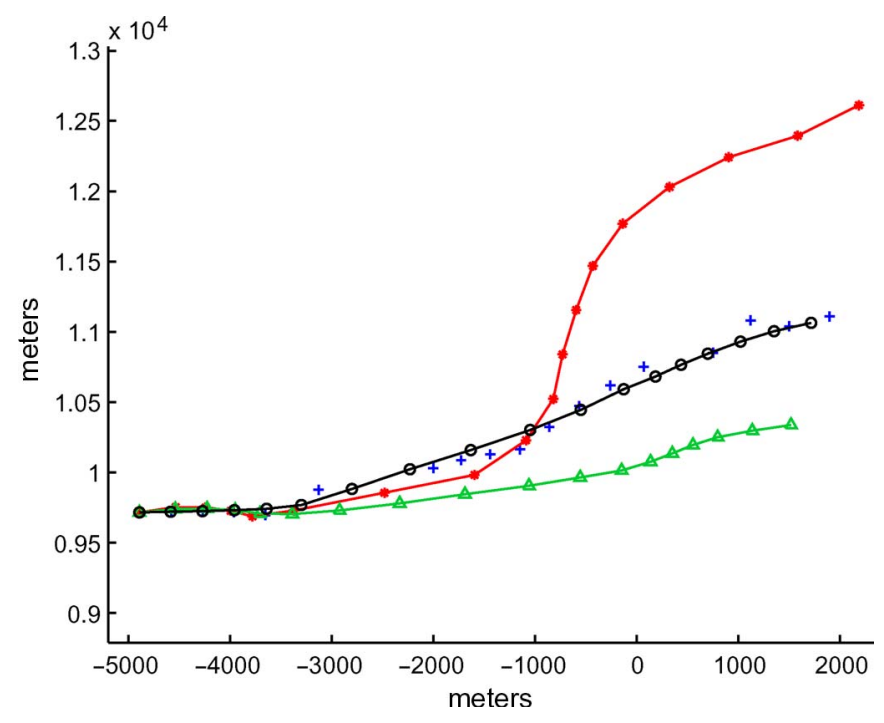

Fig. 3. (Plus marks) True locations. (Line with circles) Our estimates with the new method. (Line with triangles) Output of the traditional bootstrap filter. (Line with stars) Estimates with the EKF. As the results indicate, the new approach yields very close estimates of the true states, whereas the others weakly follow or lose track.

the source acoustic intensity, respectively. Once the acoustic intensity received by a sensor exceeds these thresholds, the sensor will be switched to the active mode and will be able to transmit the target's DOA to the fusion center. Activated sensors with the above thresholds are plotted in Fig. 4(b)-(d), respectively. As illustrated in this figure, when the threshold is set to $78 \%$ of the source intensity, only one or two sensors are in the active mode during each sampling period. This indicates that $78 \%$ is approximately the highest threshold that can be used for this particular network topology since it is assumed that at least one sensor is active during each sampling period. 


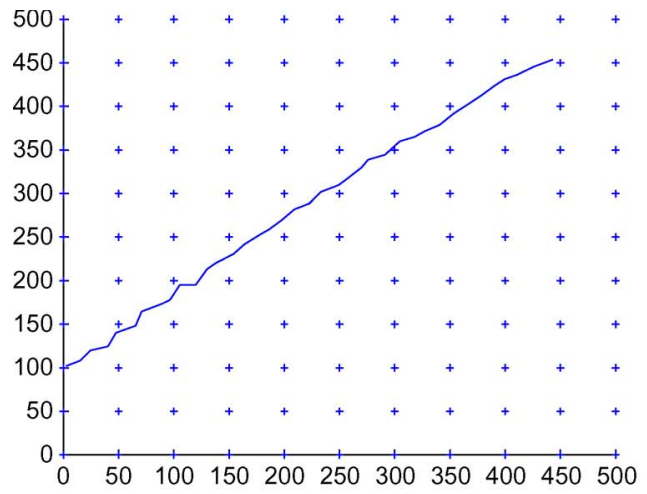

(a)

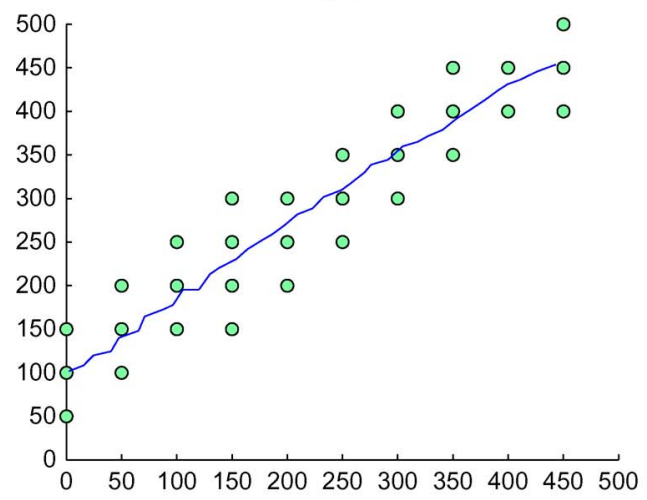

(c)

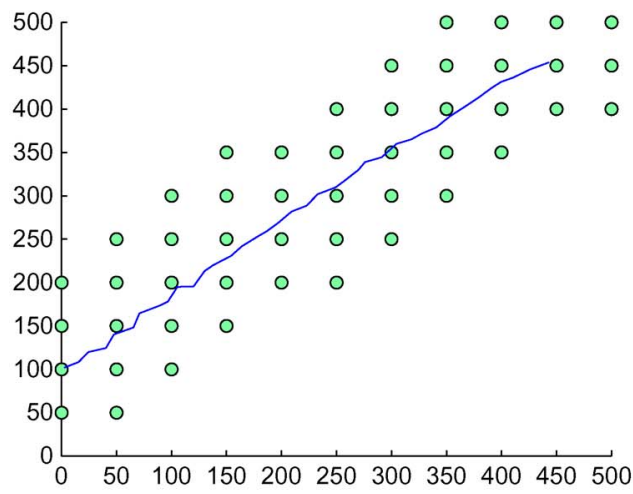

(b)

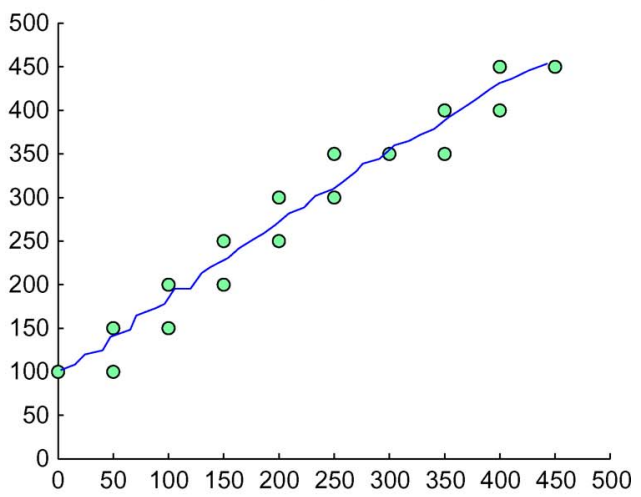

(d)

Fig. 4. (a) Grid sensor topology and the true target trajectory. (b)-(d) Activated sensors with sensor thresholds setting to $72 \%$, $75 \%$, and $78 \%$ of the source energy, respectively.

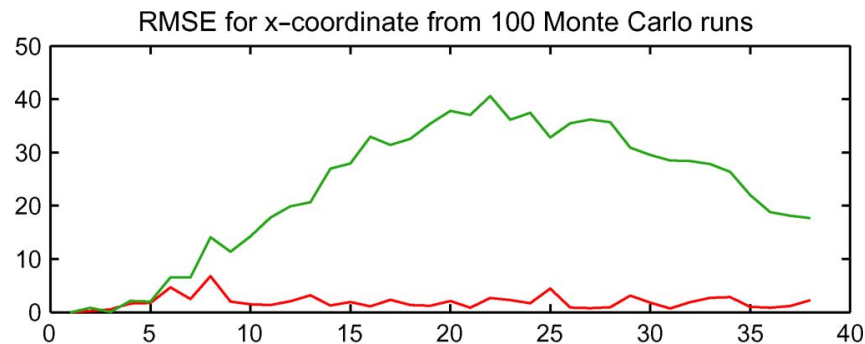

RMSE for y-coordinate from 100 Monte Carlo runs

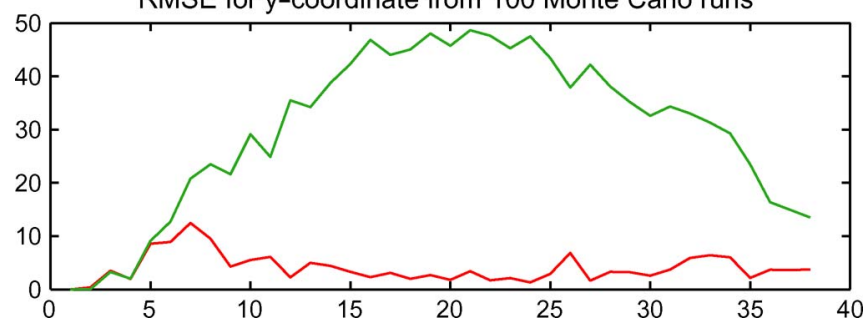

Fig. 5. RMSEs of scenario number 1. (Red line) RMSE from the SP-CPF. (Green line) RMSE from the bootstrap filter.

Next, we apply an SP-CPF and a centralized bootstrap filter (a standard $\mathrm{PF}$ ) to this problem for comparison. The sensor threshold is set to $75 \%$ of the source acoustic intensity. One hundred Monte Carlo runs are implemented to generate the statistical performance index, i.e., the root-meansquare error (RMSE). The RMSEs for the $x$-coordinate and the $y$-coordinate are shown in Fig. 5. As indicated in this figure, the SP-CPF gives very accurate estimations, whereas the
TABLE I

PERFormance INDEX OF TRACKING SCENARIO NUMBER 1

\begin{tabular}{||c|c|c|c|c|c||}
\hline \hline & \multicolumn{2}{|c|}{ RMSE for $x$} & \multicolumn{2}{c|}{ RMSE for $y$} & Time \\
\cline { 2 - 5 } & Mean & Var. & Mean & Var. & (Sec.) \\
\hline \hline SP-CPF & 1.909 & 1.693 & 4.004 & 6.978 & 2.161 \\
\hline Bootstrap & 23.180 & 154.63 & 30.060 & 223.291 & 8.109 \\
\hline \hline
\end{tabular}

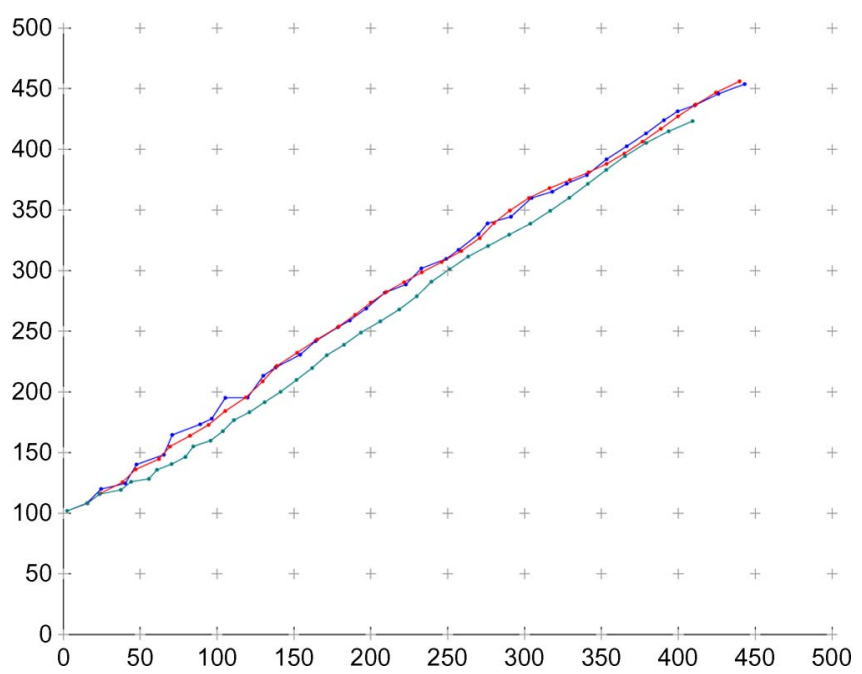

Fig. 6. Location estimations from one typical realization of scenario number 1. (Blue line) True target trajectory. (Red line) SP-CPF estimations. (Green line) Bootstrap filter estimations.

traditional bootstrap filter yields a huge estimation error. This is mainly because using the bootstrap filter takes the target state transition prior as the proposal distribution for the PF, which 

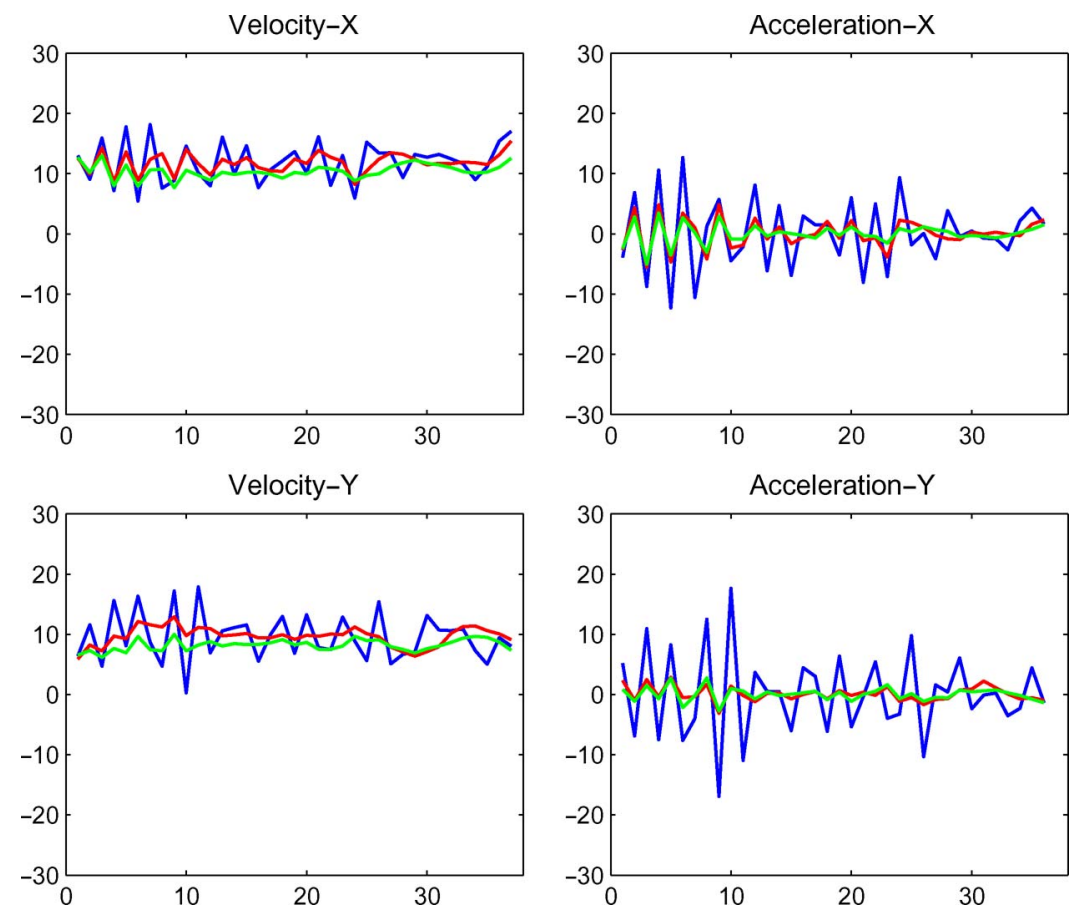

Fig. 7. Velocity and acceleration estimations from one typical realization of scenario number 1. (Blue line) True target. (Red line) SP-CPF estimations. (Green line) Bootstrap filter estimations.
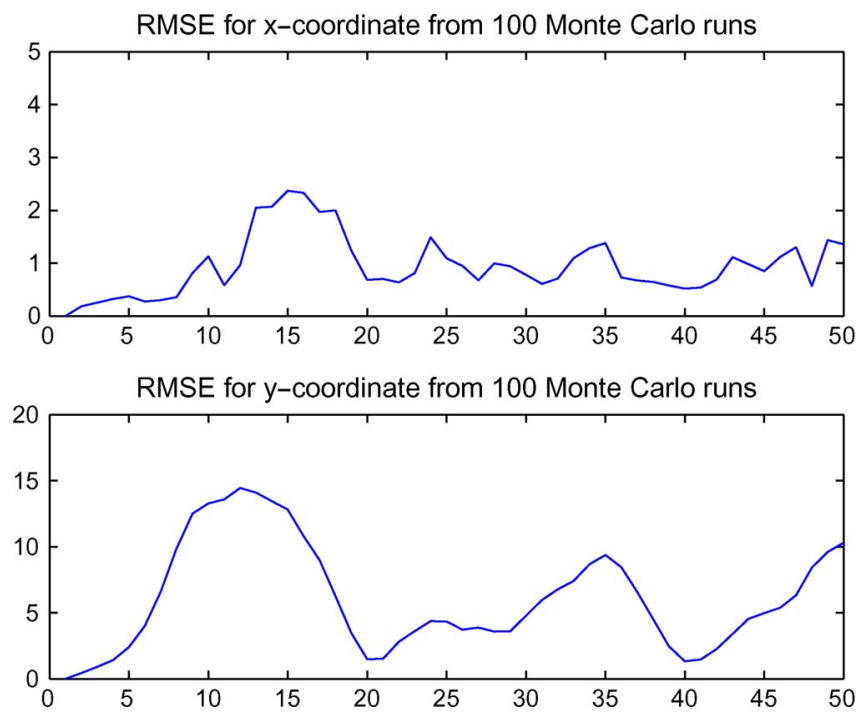

Fig. 8. RMSEs of scenario number 2.

does not include the current measurement. When the likelihood distribution is narrow with respect to the state transition prior distribution, many particles will receive negligible weights, and large estimation errors occur. The overall mean and variance of the RMSEs are shown in Table I, from which it is clear that the SP-CPF gives not only a small estimation error but a small estimation error variance as well, which means that the SP-CPF gives much more consistent estimations compared with the bootstrap filter. It should be also noted that the SP-CPF renders such good estimations with only 200 particles, whereas the bootstrap filter uses 2000 particles. The execution time for each algorithm is also provided in Table I.

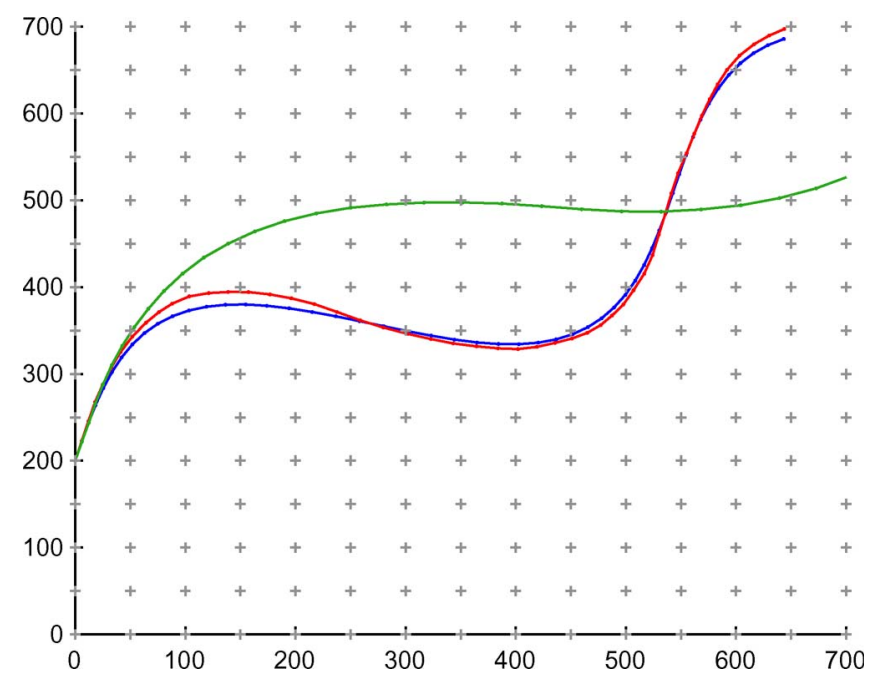

Fig. 9. Location estimations from one typical realization of scenario number 2. (Blue line) True target trajectory. (Red line) SP-CPF estimations. (Green line) Bootstrap filter estimations.

Figs. 6 and 7 illustrate a typical realization of this tracking scenario. As demonstrated in these figures, the SP-CPF algorithm keeps a close track of the target throughout the whole experiment. However, for the bootstrap filter, although it can follow the general direction of the target, it gives large estimation errors when compared with that of the SP-CPF, as shown in Fig. 6. In addition, our research also shows that when setting the threshold to $78 \%$, the SP-CPF can provide estimation results that are similar to those mentioned above, which demonstrates that this algorithm has the advantage of reducing the computation and communication load at sensor nodes, which is required to give accurate results. 

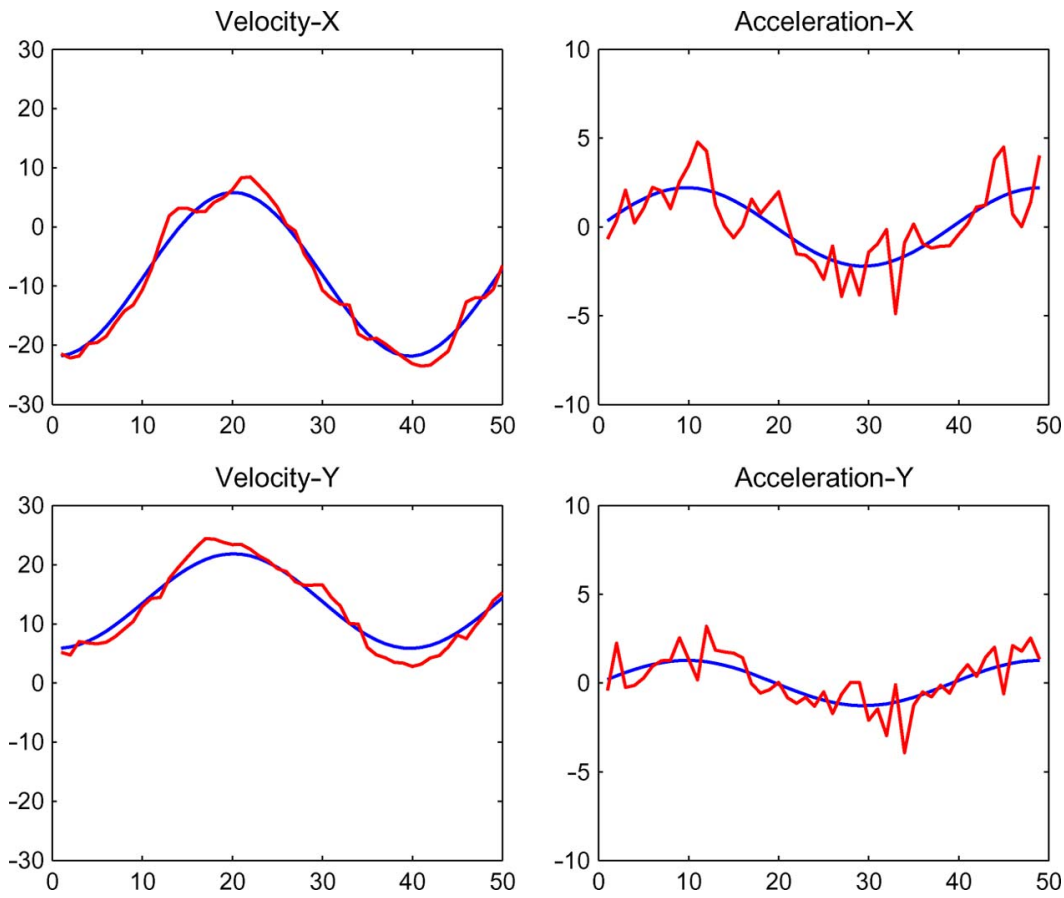

Fig. 10. Velocity and acceleration estimations from one typical realization of scenario number 2. (Blue line) True target. (Red line) SP-CPF estimations.

\section{B. Tracking Scenario Number 2}

In the second scenario, we apply the tracking algorithm to a target whose motion trajectory has two sharp coordinate turns with periodic changing velocities and accelerations. In a target-tracking community, this kind of a target is classified as a maneuvering target [27]. By definition, this implies that the target's $x$ and $y$ velocities are nonconstant as it makes a path. This aspect presents additional tracking challenges, as compared to tracking nonmaneuvering targets. Traditionally, multiple-model techniques are used to track maneuvering targets [5]. However, to test the algorithm robustness for model uncertainties, we still use the single non-maneuvering model for the SP-CPF. As in the previous example, 100 Monte Carlo runs were implemented. As shown in the experiments, the SP-CPF can keep a close track in every realization. However, the bootstrap filter loses the target in more than $80 \%$ of the 100 realizations. Due to the fact that most of the bootstrap filters lose track, we only provide the RMSE of the SP-CPF, which is shown in Fig. 8. A typical realization is shown in Figs. 9 and 10. As it is shown from this simulation, the SP-CPF closely follows the target, despite the fact that the model that is utilized here is a single non-maneuvering model. This fact demonstrates that the SP-CPF can achieve certain robustness under model uncertainties. Moreover, this robust tracking is achieved by using only 200 particles, which is a rather small sample size compared to the standard bootstrap PF that uses 2000 particles.

\section{Tracking Scenario Number 3}

In the third scenario, we investigate tracking a target with a dual-mode motion trajectory that is initially described by a constant acceleration and then followed by a constant de-

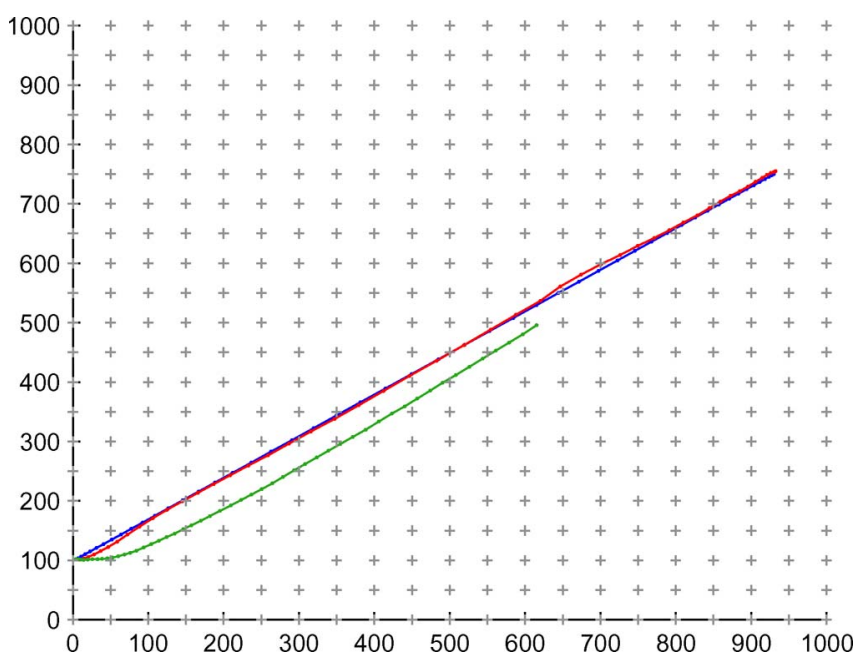

Fig. 11. Location estimations from one typical realization of scenario number 3. (Blue line) True target trajectory. (Red line) SP-CPF estimations. (Green line) Bootstrap filter estimations.

celeration. Due to the instantaneous change in the velocity and the acceleration, the target makes a significant maneuver operation, although its motion is still rectilinear. As indicated in the 100 Monte Carlo runs, the bootstrap filter can follow the general direction of the target; however, it fails to detect the instantaneous acceleration change, and, finally, it loses the target. Figs. 11 and 12 depict one realization. It is very clear in Fig. 12 that the bootstrap filter cannot detect the velocity and acceleration change. On the other hand, the SP-CPF yields very good estimates for both target locations and velocities. The RMSEs of the Monte Carlo runs are shown in Fig. 13. 

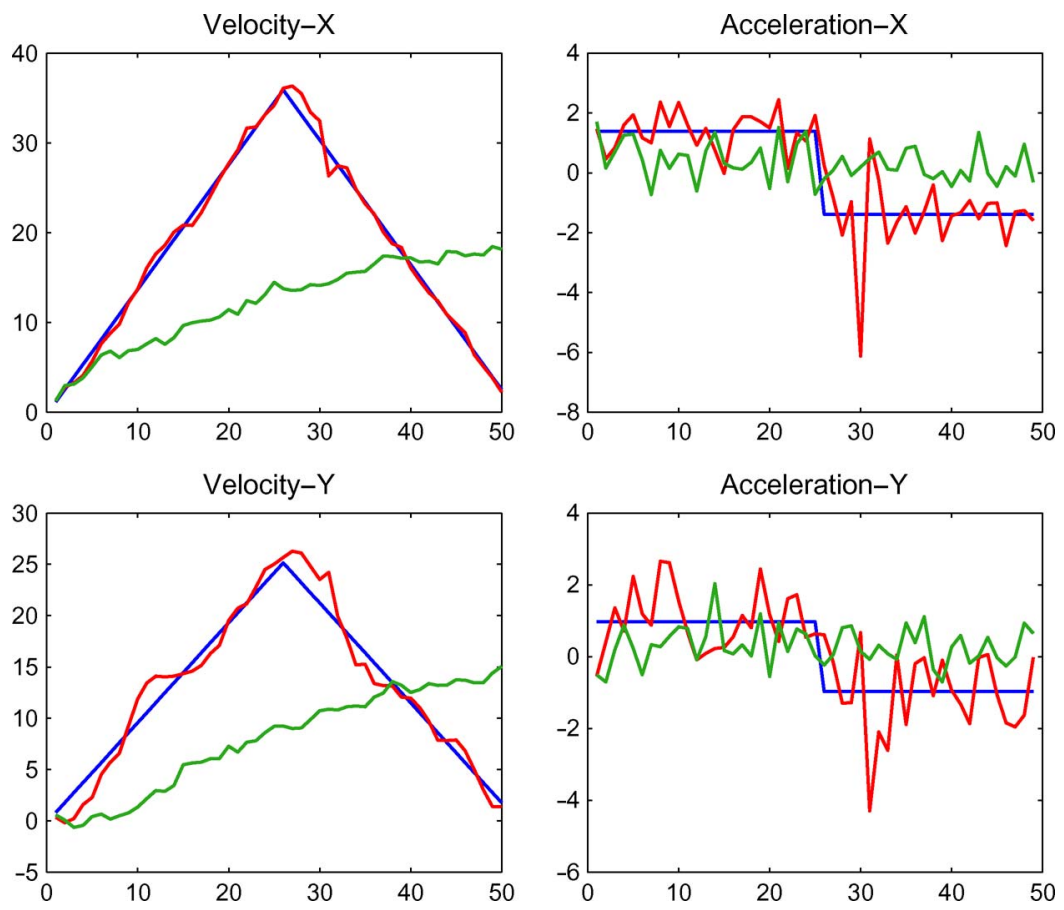

Fig. 12. Velocity and acceleration estimations from one typical realization of scenario number 3. (Blue line) True target. (Red line) SP-CPF estimations. (Green line) Bootstrap filter estimations.
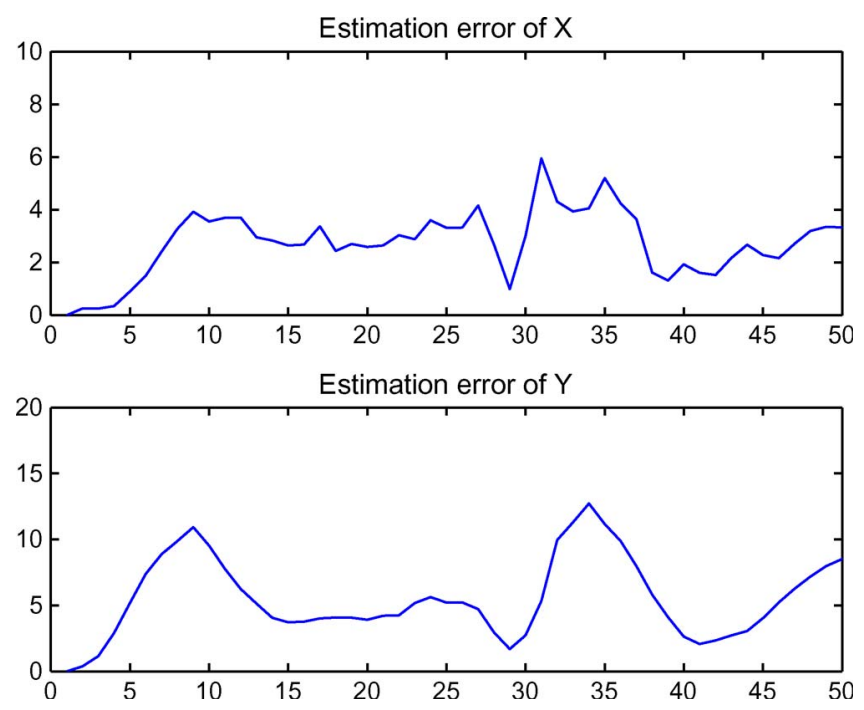

Fig. 13. RMSEs of scenario number 3.

\section{CONCLUSION}

In this paper, we have proposed a new sensor network targettracking method-based particle filtering and state partition technique. Simulation results demonstrate that this technique is capable of yielding accurate and robust estimations while reducing the computations and channel requirements in the sensor network. In particular, the dynamic model of a moving target via an acoustic sensor network has been analyzed. The network has been defined as a distributed wireless acoustic sensor network that is composed of deterministically or randomly deployed sensors, and the position of these sensors is known to the fusion center, which is either stationary or mobile. This paper has provided contributions by showing that improved sequential Monte Carlo methods can be used to efficiently generate precise estimations for targets that have complex motion patterns, and sensor nodes can be switched between the active mode and the sleep mode that is adaptively based on the requirements of power consumption and estimation accuracy.

\section{REFERENCES}

[1] F. Zhao and L. Guibas, Wireless Sensor Networks: An Information Processing Approach. San Francisco, CA: Morgan Kaufmann, 2004.

[2] I. Akyildiz, S. Weilian, Y. Sankarasubramaniam, and E. Cayirci, "A survey on sensor networks," IEEE Commun. Mag., vol. 40, no. 8, pp. 102-114, Aug. 2002.

[3] K. Lee and M. Reichardt, "Open standards for homeland security sensor networks," IEEE Instrum. Meas. Mag., vol. 8, no. 5, pp. 14-21, Dec. 2005.

[4] Y. Zhai, M. Yeary, and J.-C. Noyer, "Target tracking in a sensor network based on particle filtering and power-aware design," in Proc. IEEE IMTC, Apr. 2006, pp. 1988-1992.

[5] Y. Bar-Shalom, Multitarget Multisensor Tracking: Advanced Applications. Boston, MA: Artech House, 1990.

[6] F. Martinerie, "Data fusion and tracking using HMMs in a distributed sensor network," IEEE Trans. Aerosp. Electron. Syst., vol. 33, no. 1, pp. 11-28, Jan. 1997.

[7] X. Sheng and Y.-H. Hu, "Energy based acoustic source localization," in Proc. Inf. Process. Sensor Netw., Apr. 2003, pp. 285-300.

[8] X. Sheng and Y.-H. Hu, "Sequential acoustic energy based source localization using particle filter in a distributed sensor network," in Proc. IEEE ICASSP, May 2004, pp. 972-975.

[9] W. Zhang and G. Cao, "DCTC: Dynamic convoy tree-based collaboration for target tracking in sensor networks," IEEE Trans. Wireless Commun., vol. 3, no. 5, pp. 1689-1701, Sep. 2004.

[10] M. Orton and W. Fitzgerald, "A Bayesian approach to tracking multiple targets using sensor arrays and particle filters," IEEE Trans. Signal Process., vol. 50, no. 2, pp. 216-223, Feb. 2002.

[11] M. Coates, "Distributed particle filters for sensor networks," in Proc. IPSN, Apr. 2004, pp. 99-107.

[12] C. Kreucher, K. Kastella, and A. Hero, "Multi-target sensor management using Alpha-divergence measures," in Proc. IPSN, Apr. 2003, pp. 209-222. 
[13] X. Sheng and Y.-H. Hu, "Distributed particle filters for wireless sensor network target tracking," in Proc. IEEE ICASSP, 2005, pp. 845-848.

[14] N. Patwari et al., "Locating the nodes: Cooperative localization in wireless sensor networks," IEEE Signal Process. Mag., vol. 22, no. 4, pp. 54-69, Jul. 2005.

[15] S. Gezici et al., "Localization via ultra-wideband radios: A look at positioning aspects for future sensor networks," IEEE Signal Process. Mag., vol. 22 , no. 4, pp. 70-84, Jul. 2005.

[16] G. Sun et al., "Signal processing techniques in network-aided positioning: A survey of state-of-the-art positioning designs," IEEE Signal Process. Mag., vol. 22, no. 4, pp. 12-23, Jul. 2005.

[17] B. Sadler, R. Kozick, and L. Tong, "Multi-modal sensor localization using a mobile access point," in Proc. IEEE ICASSP, Mar. 2005, vol. 4, pp. 753-756.

[18] A. Doucet, N. de Freitas, and N. Gordon, Sequential Monte Carlo Methods in Practice. New York: Springer-Verlag, 2001.

[19] P. Djurić et al., "Particle filtering," IEEE Signal Process. Mag., vol. 20, no. 5, pp. 19-38, Sep. 2003

[20] M. Arulampalam, S. Maskell, N. Gordon, and T. Clapp, "A tutorial on particle filters for online nonlinear/non-Gaussian Bayesian tracking," IEEE Trans. Signal Process., vol. 50, no. 2, pp. 174-188, Feb. 2002.

[21] Y. Zhai and M. Yeary, "A novel nonlinear state estimation technique based sequential importance sampling and parallel filter banks," in Proc. IEEE Int. Conf. Control Appl., Aug. 2005, pp. 1606-1611.

[22] L. Kinsler et al., Fundamentals of Acoustics. New York: Wiley, 1982.

[23] J. Kang, Acoustics of Long Spaces: Theory and Design Practice. London, U.K.: Thomas Telford, 2002.

[24] D. Lainiotis and P. Papaparaskeva, "Efficient algorithms of clustering adaptive nonlinear filters," IEEE Trans. Autom. Control, vol. 44, no. 7, pp. 1454-1459, Jul. 1999.

[25] D. G. Lainiotis and P. Papaparaskeva, "A new class of efficient adaptive nonlinear filters (ANLF)," IEEE Trans. Signal Process., vol. 46, no. 6, pp. 1730-1737, Jun. 1998.

[26] B. Ristic, S. Arulampalam, and N. Gordon, Beyond the Kalman Filter: Particle Filters for Tracking Applications. Norwood, MA: Artech House, Feb. 1, 2004.

[27] X. Li and V. Jilkov, "Survey of maneuvering target tracking. Part I. Dynamic models," IEEE Trans. Aerosp. Electron. Syst., vol. 39, no. 4, pp. 1333-1364, Oct. 2003.

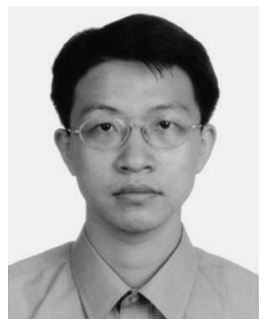

Yan Zhai (S'03-M'07) received the B.S. degree from Tsinghua University, Beijing, China, the M.S. degree in mechanical engineering from the Oklahoma State University, Stillwater, and the Ph.D. degree in electrical engineering from The University of Oklahoma, Norman, in 1998, 2002, and 2007, respectively.

$\mathrm{He}$ is currently with Schlumberger Technology Center, Sugar Land, TX. His primary research is in the area of stochastic signal processing with a focus on developing sequential Monte Carlo algorithms and applying them to nonlinear state estimation applications. He has written a variety of papers and has served as a reviewer for several national and international conferences and journals.

Dr. Zhai was a recipient of the Sooner Heritage Scholarship from The University of Oklahoma. He is a member of Sigma Xi and Tau Beta Pi.

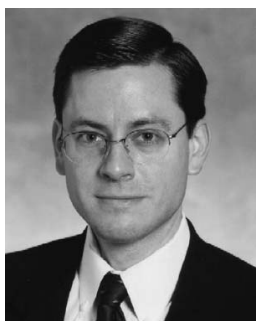

Mark B. Yeary (S'95-M'00-SM'03) received the B.S. (honors), M.S., and Ph.D. degrees from the Department of Electrical Engineering, Texas A\&M University (TAMU), College Station, in 1992, 1994, and 1999 , respectively.

As a student at TAMU, he was a Charter Member and Officer of the Engineering Scholars Program and a recipient of the Dean's Outstanding Student Award. In 1995, he was with International Business Machines (IBM), Austin, TX, as a member of a microprocessor development team. As a graduate student, he served as a Teaching and Research Assistant. Following his graduation in 1999, he continued to be a member of the DSP group and a Lecturer with the Department of Electrical Engineering, TAMU. He received the Outstanding Teaching Assistant award from the local IEEE Student Chapter two years in a row and was nominated to be a National Science Foundation/Frontiers in Education 1998 New Faculty Fellow. Since Fall 2002, he has been an Assistant Professor with the School of Electrical and Computer Engineering at The University of Oklahoma, Norman. His research, teaching, and consulting interests are in the areas of customized embedded DSP systems and digital signal processing as applied to radar signal processing, digital communications, image processing, adaptive filter design, and real-time systems. In addition, he has spent six summers (2002-2007) at Raytheon near Dallas, TX, as faculty researcher. His applied signal processing contributions there are many and include the design of an all-digital system-on-a-chip scheme for a KA band radar and various target tracking algorithm developments.

Dr. Yeary is a member of the Tau Beta Pi and Eta Kappa Nu honor societies. $\mathrm{He}$ received the IEEE Outstanding Young Engineer Award from the I\&M Society in 2005, and its citation read "for contributions to radar systems measurements." He has served on numerous national and international review panels. For instance, he has served as a Technical Committee Member (TPC) for IMTC in the past and is currently on the IMTC's TPC for 2008

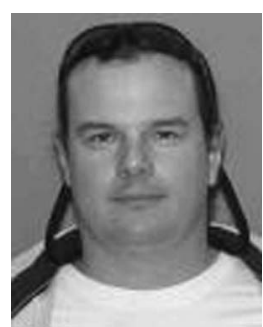

Joseph P. Havlicek (S'84-M'88-SM'98) received the B.S. and M.S. degrees from Virginia Polytechnic Institute and State University, Blacksburg, and the $\mathrm{Ph} . \mathrm{D}$. degree from the University of Texas, Austin, all in electrical engineering.

From 1984 to 1987, he was a Software Developer with Management Systems Laboratories, Blacksburg. In 1993, he was with International Business Machines, Austin, where he was affiliated with Ralph Kirkley Associates. From 1987 to 1997, he was with the Naval Research Laboratory, Washington, DC, where, from 1987 to 1989, he was associated with SFA, Inc. In 1997, he joined School of Electrical and Computer Engineering, The University of Oklahoma, Norman, where he is currently a Professor. His research interests include signal, image, and video processing and intelligent transportation systems.

Dr. Havlicek has been a member of the organizing committee of the IEEE Southwest Symposium on Image Analysis and Interpretation since 1998 and served as the Technical Program Cochair in 2004, 2006, and 2008. He also served as the Publications Chair on the organizing committee of the IEEE International Conference on Image Processing in 2007. He was a recipient of the Department of the Navy Award of Merit for Group Achievement in 1990 for his work. He is a member of Tau Beta Pi, Phi Kappa Phi, and Eta Kappa $\mathrm{Nu}$

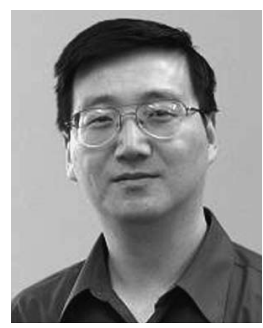

Guoliang Fan (S'97-M'01-SM'05) received the B.S. degree in automation engineering from Xi'an University of Technology, Xi'an, China, the M.S. degree in computer engineering from Xidian University, Xi' an, and the Ph.D. degree in electrical engineering from the University of Delaware, Newark, in 1993, 1996, and 2001, respectively.

From 1996 to 1998, he was a Graduate Assistant with the Department of Electronic Engineering, The Chinese University of Hong Kong, Shatin, Hong Kong. Since 2001, he has been with School of Electrical and Computer Engineering, Oklahoma State University (OSU), Stillwater, where he is currently an Associate Professor. His research interests include image processing, computer vision, biomedical imaging, and remote sensing applications.

Dr. Fan was awarded First Prize at the 1997 IEEE Hong Kong Section Postgraduate Student Paper Contest and First Prize at the 1997 IEEE Region 10 (Asia-Pacific) Postgraduate Paper Contest. He was a recipient of the National Science Foundation CAREER award in 2004 and of the Halliburton Excellent Young Teacher Award and Halliburton Outstanding Young Faculty Award in 2004 and 2006, respectively, from OSU. 